\title{
Optimization for Maximum Nonlinear Buckling Load and Topics on Imperfection of Latticed Shells
}

\author{
Pei-Shan Chen \\ Department of Civil Engineering and Architecture \& Graduate School, Hachinohe Institute of Technology, Aomori 031-8501, Japan
}

\begin{abstract}
The present paper represents comparison of continuum shells and latticed shells with qualitative analysis. For shells, the mechanical characteristics in the two perpendicular directions are continuous and related to each other, and any change in thickness will result in change in stiffness in any direction. In latticed shells, members are discrete and stiffnesses in two mutually perpendicular directions are discontinuous and independent of each other. Therefore, sensitivity of geometrical imperfection for buckling of latticed shells should be different from that of continuum shells. The author proposes a shape optimization method for maximum buckling load of a latticed shell. A single layer latticed dome is taken as a numerical example, and the results show that the buckling load parameter for full area loading case increases $32.75 \%$ compared to that of its initial shape. Furthermore, the numerical example demonstrates that an optimum latticed shell with maximum buckling load, unlike an optimum continuum shell, may not be sensitive to its geometrical imperfection.
\end{abstract}

Key words: Latticed shell, shape optimization, buckling load, imperfection, sensitivity analysis, state of the art, space frame.

\section{Introduction}

Shape optimization for maximum buckling load of latticed shells (single layer space frames), especially maximizing the nonlinear buckling load, is still a citadel in the fields of mechanical analysis and structural engineering [1]. Furthermore, shape optimization against buckling is always followed by sensitivity analysis of initial geometrical imperfection, which is an attractive theme for all practical purposes. Papers on optimization for maximum buckling load of continuum shells have been published in the past issues, and many of them pointed out that an optimum shell is very sensitive to its geometrical imperfection which causes buckling load reduction [2]. Furthermore, latticed shells are always treated as continuum shells for engineering approximation. Therefore, mechanical manners for continuum shells may easily be applied to latticed shells, and associated with that an optimum latticed shell with maximum buckling load may be very sensitive to its initial

Corresponding author: Pei-Shan Chen, Dr., Eng., professor, research fields: structural design and structural analysis. E-mail: chen@hi-tech.ac.jp. imperfection as a continuum shell does. However, this is not the final conclusion. It is necessary to detect the mechanical differences between continuum shells and latticed shells, and consequently come to the conclusion that if an optimum latticed shell must be sensitive to its imperfection and causes buckling load reduction.

Therefore, the present paper attempts to compare the mechanical characteristics of continuum shells and latticed shells with a qualitative analysis manner, and proves that the optimum shape of a latticed shell with maximum buckling load may not be sensitive to its geometrical imperfection. Hence, the author proposes a shape optimization for maximum buckling load of a latticed shell, and demonstrates its buckling load reduction caused by geometrical imperfection.

\section{Imperfection of Continuum Shells and Latticed Shells}

Pioneering works on the shells and space frames for buckling load reduction due to geometrical imperfection have been issued. Ramm and Wall [2] indicate that shells may be extremely parameter 
sensitive especially if it is built with super-symmetry shape or high stiffness [2]. It was shown by Reitinger and Ramm [3] that more insensitive structure can be obtained by maximizing the buckling load of the imperfection shapes instead of the perfect shape. In addition, they indicated that mechanically sensitive problems will be and need to be also numerically sensitive for numerical analyses. On the other hands, Osaki and Ikeda [4] explained that in the case of the hill-top branching, the buckling loads of imperfect systems are piecewise linear functions of imperfection parameter, and showed that its imperfection sensitivity is lower than the case where a bifurcation point exists independently.

Thought latticed shells are similar to continuum shells in out form and always treated with theories of shells, their nonlinear behaviors are not the same. Therefore, sensitivity of imperfection for buckling of continuum shells should be different from that of latticed shells. For a good understanding of the mechanical differences between continuum shells and latticed shells, the author compares the mechanical characteristics of the two in Table 1. For a latticed shell shown in the table, the stiffness in same direction, $K_{x}$ and $K_{x}$ ' for example, may be discontinued, and the stiffnesses in different directions $K_{x}$ and $K_{y}$, may be completely independent. On the other hand, changes in thickness of a continuum shell must lead to the changes in both $K_{x}$ and $K_{y}$, as well as bending stiffness. Furthermore, an ideal shell requires thin thickness for small bending, and then its buckling load is sensitive to its shape parameters.

Because a latticed shell may be built with anisotropic and discontinued stiffness, it may be designed with suitable members in latitude direction to stiffen the members in meridian direction for high buckling resistance. Such a significant effect is very difficult to be obtained for a continuum shell, unless it is built by orthotropic material or stiffeners. Kassegne and Reddy [5] indicated that stiffeners of a shell wall, can increase the critical load (the buckling load), and multiple stiffeners increase the critical load very significantly. More researches by Yamada and Yano [6] indicated that the buckling behavior of a shell with orthotropic material may not be sensitive to its shape imperfection comparing with the isotropic ones.

However, the numerical example in the present paper, as will be shown in Section 4, demonstrates that an optimum shape with maximum buckling load of a latticed shell may not be sensitive to its shape imperfection. Therefore, the author promotes an inference in the following: For a latticed shell as well as a shell with orthotropic material and suitable stiffeners, its optimum shape with maximum buckling load may not be sensitive to its geometrical imperfection.

Table 1 Comparison of mechanical characteristics between shells and latticed shells.

\begin{tabular}{|l|l|}
\hline Shells & Latticed shells (single layer space frames) \\
\hline Built by thin continuum in curvature & $\begin{array}{l}\text { Built by discrete members } \\
\text { The stiffness } K_{x} \text { and } K_{y} \text { are continue respectively and related to } K x \text { and } K y \text { are discontinue respectively and } \\
\text { each other }\end{array}$ \\
\hline Changes in thickness lead to the changes in bending stiffness & $\begin{array}{l}\text { Member section changes cause slight changes in global bending } \\
\text { stiffness }\end{array}$ \\
\hline
\end{tabular}


However, this inference is a challenged topic to be demonstrated by theoretical and experimental manners.

\section{Shape of Maximum Buckling Load}

In order to demonstrate the characteristics of latticed shells with optimum shape of maximum buckling load, the author proposes an optimization method and shows a numerical example consequently. Though the researches on optimization for maximum buckling load of thin shells as well as laminated walls have been carried out [7, 8], the author and his co-author(s) proposed a strategy to find the optimum shape of maximum buckling load of latticed shells. The proposed optimization expresses the buckling load as an explicit function (objective function), and employs the sensitivity/derivatives of the objective function with respect to the design parameters $[9,10]$, as will be demonstrated in the next parts.

\subsection{Formulation of the Optimization}

Because the proposed optimization targets on nonlinear buckling load, it is necessary to solve the nonlinear equations of equilibrium and search for the critical point on the equilibrium path for every step of the optimization process. In general, a nonlinear structural analysis is based on the incremental equation shown below:

$$
\mathbf{K d}=\lambda \mathbf{f}
$$

where, $\mathbf{K}$ is the tangential stiffness matrix, $\mathbf{d}$ is the vector of changes in displacements $\mathbf{D}=\left\{D_{i}\right\}, \mathbf{f}$ is the load mode vector, and $\lambda$ is the change in load parameter $\Lambda=\sum \lambda^{u} \quad(u$ indicates the incremental step of the nonlinear analysis). For a common analysis procedure, the load mode vector keeps constant, and its coefficient, the load parameter, is increased step by step by the iteration. Many numerical techniques, such as Riks-Method, load incremental and displacement incremental methods are developed to find the equilibrium path (load-displacement curve) [11, 12], and the buckling point can be obtained as a limit point or a bifurcation point in the load-displacement curve.

For the convenience of the mathematic analysis, the load parameter can be expressed as an explicit function by following manners. Firstly, a vector $\mathbf{b}$ can be defined by the following equations:

$$
\mathbf{b}^{\top} \mathbf{f}=1, \quad b_{i}=\left\{\begin{array}{l}
0, \forall f_{i}=0 \\
\frac{1}{\mathrm{~N}} f_{i}^{-1}, \forall f_{i} \neq 0
\end{array}\right.
$$

where, $N$ is the number of nonzero elements of the load mode vector $\mathbf{f}=\left\{f_{i}\right\}$. Then, an explicit function of the load parameter at incremental step $u$ can be obtained as the following equation:

$$
\lambda^{u}=\mathbf{b}^{\top} \mathbf{K}^{u} \mathbf{d}^{u}
$$

A structure should be able to support multi-loading cases. In addition, the buckling capability as well as the optimum shape depends on the loading cases (loading modes). Therefore, weighting function method is adopted to establish the objective function for multi-loading modes as that expressed below. For every loading mode, ${ }_{i} \mathbf{f}(i=1$, $2, \ldots, l)$, vector ${ }_{i} \mathbf{b}(i=1,2, \ldots, l)$ can be obtained by Eq. (2), and the load parameter can also be obtained by Eq. (3) as well. If the buckling point due to ${ }_{i} \mathbf{f}$ is found at step $s$ of the nonlinear structural analysis, the total load parameters ${ }_{i} \Lambda$ can be obtained by following equation:

$$
{ }_{i} \Lambda={ }_{i} \mathbf{b}^{\top} \sum_{u=1}^{\mathrm{S}}{ }_{i} \mathbf{K}^{u}{ }_{i} \mathbf{d}^{u} ; \quad(i=1,2, \cdots, l)
$$

Then, the objective function of the optimization is in form:

$$
F=\sum_{i=1}^{l} w_{i}^{i} \Lambda
$$

where, $w^{1}, \ldots, w^{l}, \sum_{i} w^{i}=1$ are weighting factors for loading modes, which are determined by the designers of the latticed shell. Hence, the optimization for multi-loading cases can be formulated as: 


$$
\left\{\begin{array}{l}
\text { Maximize } F=\sum_{i=1}^{l} w^{i}{ }_{i} \Lambda \\
\text { Subject to: } \\
h_{j}(\mathbf{X}, \mathbf{U})=0 ; \quad(j=1, \ldots, s) \\
g_{k}(\mathbf{X}, \mathbf{U}) \leq 0, \quad(k=1, \ldots, t)
\end{array}\right.
$$

where, $\mathbf{X}$ is the vector of the design parameters of joint coordinates with $n$ elements, and $\mathbf{U}$ the other design parameters vector with $m$ elements. Eq. (7) indicates the equality constraints, Eq. (8) indicates the inequality constraints, and these conditions make a constraint space of the optimization. In the optimization, $n+m$ design parameters of $\mathbf{X}$ and $\mathbf{U}$ may be changed to reach the maximum of the objective function $F$ and buckling load parameters ${ }_{i} \Lambda$ $(i=1,2, \ldots, l)$ as well.

\subsection{Solution Method for the Optimization}

In the present paper, the author attempts to solve the problem by GRG (general reduced gradient) method [13], which is efficient to find the optima in the vicinity of the initial design parameters. In GRG, the incremental direction of the objective function is defined by the derivative of the objective function with respect to the design parameters. The derivative of the objective Eq. (6) with respect to design parameter $\boldsymbol{X}$ and $\boldsymbol{U}$ can be obtained in the form of:

$$
\begin{gathered}
\dot{\Lambda}_{, i}=\dot{\mathbf{b}}_{, i}^{\top} \sum_{u=1}^{T} \mathbf{K}^{u} \mathbf{d}^{u}+\mathbf{b}^{\top} \sum_{u=1}^{T} \dot{\mathbf{K}}_{, i}^{u} \mathbf{d}^{u} \\
=\Lambda \dot{\mathbf{b}}_{, i}^{\top} \mathbf{f}+\mathbf{b}^{\top} \sum_{u=1}^{T} \dot{\mathbf{K}}_{, i}^{u} \mathbf{d}^{u} \\
(i=1,2, \ldots n+m)
\end{gathered}
$$

In fact, the design parameters may not be coordinates of joints, but sub geometrical design parameters such as diameters, rises, lengths, etc.. The sub geometrical design parameters $\mathbf{q}=\left(q_{1}, q_{2}, \ldots, q_{r}\right)$ must be related to $\mathbf{X}$ and $\mathbf{U}$ by a suitable function $\mathbf{X}$ as following:

$$
(\mathbf{X}, \mathbf{U})=X\left(q_{1}, q_{2}, \ldots, q_{r}\right)
$$

Hence, the incremental direction of the objective function with respect to the sub geometrical design parameters is in the form:

$$
\Delta=\mathbf{J}(\nabla F)
$$

where, $\nabla=\left(\frac{\partial}{\partial x_{1}}, \ldots, \frac{\partial}{\partial x_{n}}, \frac{\partial}{\partial U_{1}}, \ldots, \frac{\partial}{\partial U_{m}}\right)^{\top}, \mathbf{J}$ is the Jacobian matrices for the conversion Eq. (10). In order to reach the maximum of the objective function $F$, the sub geometrical design parameters should be changed in the following form:

$$
\mathbf{q}^{k+1}=\mathbf{q}^{k}+\alpha \Delta^{k}
$$

where, $k$ is the step number of the optimization iteration and $\alpha$ a suitable step length. For every step

$$
\Lambda^{k+1}>\Lambda^{k}>\Lambda^{k-1}
$$

is expected, so that the buckling load can become bigger and bigger. However, the Kuhn and Tucker condition should be checked for whether an optimum point is reached.

\section{Numerical Analysis Example}

\subsection{The Example}

A single layer space frame shown in Fig. 1 is taken as the example for the numerical analyses. It is a dome of revolution with a definite rise and a rigid structure at the top, because such a top structure can resist dome buckling and makes a good connection between members at the top. Then, the joint coordinates and member sections can be found by the present optimization method.

The initial shape of the dome is a truncated sphere with $H=300 \mathrm{~cm}$ in rise and $R=1,500 \mathrm{~cm}$ in radius (Fig. 2). The radius of the rigid top structure is $r=500$ $\mathrm{cm}$. The dome is constituted by pipes with $3.5 \mathrm{~cm}$ in

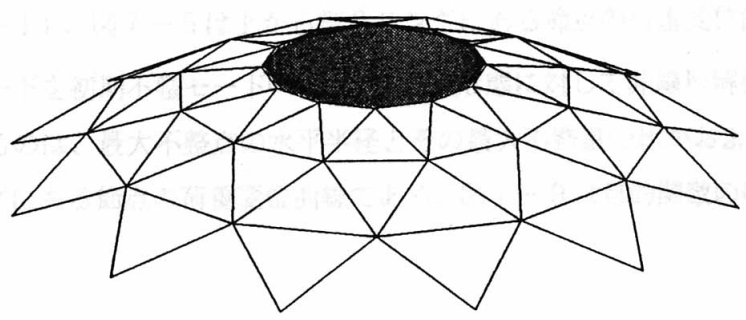

Fig. 1 Numerical analysis example. 
diameter and $0.7 \mathrm{~cm}$ in thickness as the initial dimensions of the members. The material is assumed to be a complex one with an elastic modulus of $4.91 \times$ $10^{4} \mathrm{~N} / \mathrm{mm}^{2}$ and a shear modulus of $1.27 \times 10^{4} \mathrm{~N} / \mathrm{mm}^{2}$. The elements of the load mode vector, $\mathbf{f}=\left\{f_{i}\right\}$, are concentrated loads applying at the joints, which are calculated by a vertical distribution load of $1.0 \times 10^{-4}$ $\mathrm{N} / \mathrm{mm}^{2}$. Therefore, the load mode is a function of the joint coordinates. The situation of full area loading and half area loading are shown in Fig. 3.

The analysis cases:

Case 1 (full area loading): Geometrical parameters to be found are $H 1, H 2, R 1, R 2$, but $H, R$ and $r$ are kept constant. All the member sections are kept constant as their initial values;

Case 2 (full and half area loading): Geometrical parameters to be found are $H 1, H 2, R 1, R 2$ and the member radiuses $r_{1}, r_{2}, r_{3}, r_{4}, r_{5}$. Constant parameters are $H, R, r$ and all the pipe thicknesses.

The geometrical parameters in the two cases are independent variables and no equality constraints are introduced. As an economic condition, an inequality constraint is adopted in the form: the structural volume is less than or equal to $660,000 \mathrm{~cm}^{3}$, while the initial structural volume is $614,000 \mathrm{~cm}^{3}$.

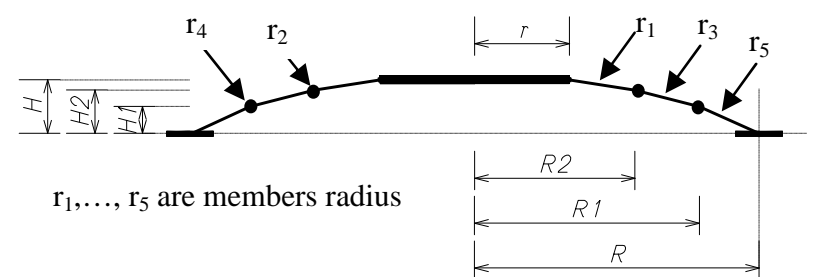

Fig. 2 The design parameters of the latticed dome.

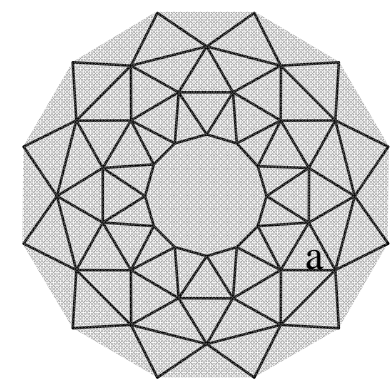

(a) Full area loading mode

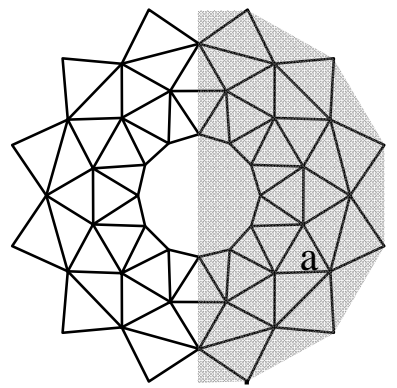

(b) Half area loading mode
Fig. 3 The loading modes.

\subsection{The Result of Case 1}

Comparison of the initial shape with the optimum shape is shown in Fig. 4. Table 2 shows the changes in the design parameters.

It shows that the joints of the dome should be changed inside its initial shape (a truncated sphere) for a bigger buckling load. Fig. 5 shows the load-displacement curve, and bifurcation buckling appearing at a point of second ring of the dome where the position of point "a" is indicated in Fig. 3. By the present optimization, the buckling load for full area loading mode increased $18.42 \%$ compared to that of its initial shape, which is a result with changes in dome curvature only.

\subsection{The Result of Case 2}

Case 2 is an optimization due to multi-loading modes.

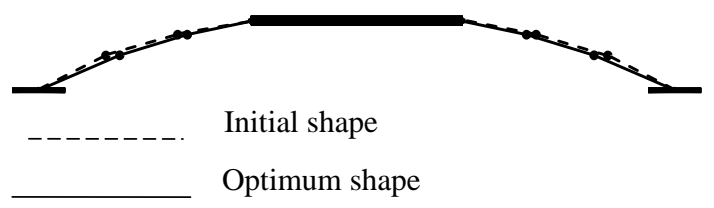

Fig. 4 Changes in the shape.

Table 2 The changes in design parameters (unit: $\mathbf{c m}$ ).

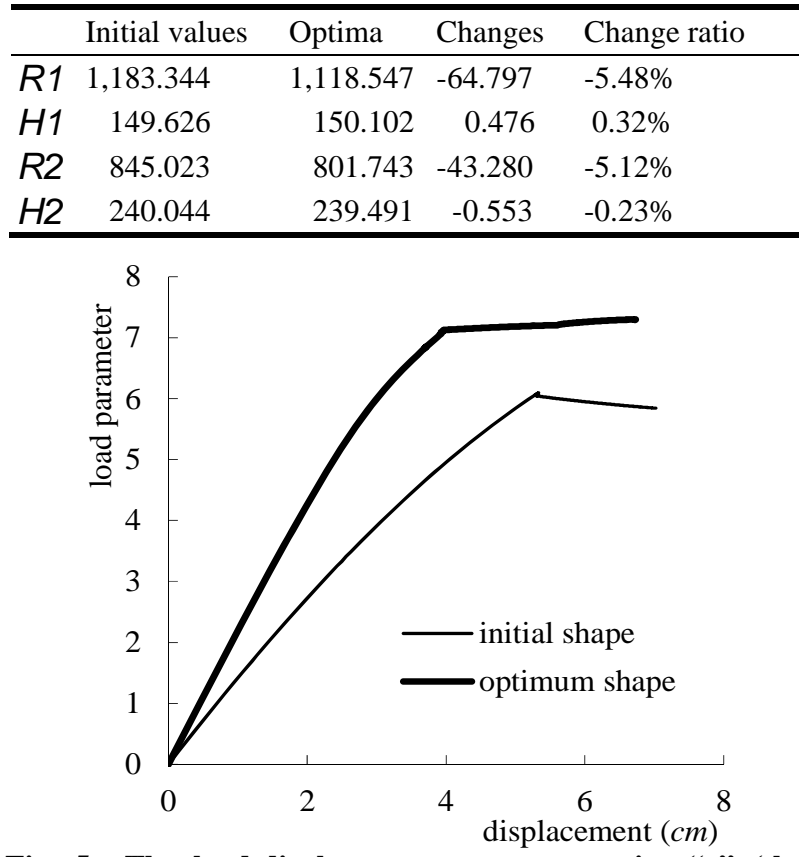

Fig. 5 The load-displacement curves at point "a" (the position of "a" is shown in Fig. 3). 
Fig. 6 shows the convergence of the optimization procedure. Table 3 lists the changes in design parameters. It is found that the member radiuses in meridian direction, $\mathrm{r}_{1}, \mathrm{r}_{3}, \mathrm{r}_{5}$, have been increased, but that in latitude direction, $r_{2}, r_{4}$, appeared very slight changes. Therefore, the stiffness in meridian direction of a latticed shell should be increased for high buckling resistance, as that indicated in Section 2.

Figs. 7 and 8 show the load-displacement curve at a point of second ring of the dome (point "a" is indicated in Fig. 3). Comparing with the initial shape, the buckling load for full area loading mode increases $32.75 \%$, and that for half loading mode increases $27.61 \%$.

Fig. 9 shows that the structural volume changes within the constraint space.

\subsection{Buckling Load Due to the Imperfection}

By this numerical example, comparisons of the buckling load reduction due to geometrical imperfection between optimum shape and initial shape are carried out. Fig. 10 shows the load-displacement

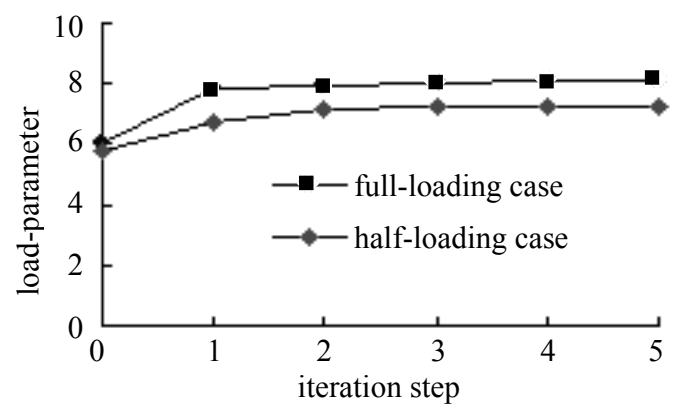

Fig. 6 The convergence of the optimization.

Table 3 The changes in design parameters (unit: $\mathrm{cm}$ ).

\begin{tabular}{lrrrl}
\hline & \multicolumn{2}{c}{ Initial values Optima } & Changes & Ratio of changes \\
\hline$R 1$ & $1,183.344$ & $1,181.245$ & -2.099 & $-0.177 \%$ \\
$H 1$ & 149.626 & 149.490 & -0.136 & $-0.091 \%$ \\
$R 2$ & 845.023 & 845.150 & 0.127 & $0.015 \%$ \\
$H 2$ & 240.044 & 242.755 & 2.711 & $1.129 \%$ \\
$\mathrm{r}_{1}$ & 3.500 & 3.605 & 0.105 & $3.00 \%$ \\
$\mathrm{r}_{2}$ & 3.500 & 3.499 & -0.001 & $-0.028 \%$ \\
$\mathrm{r}_{3}$ & 3.500 & 3.660 & 0.160 & $4.571 \%$ \\
$\mathrm{r}_{4}$ & 3.500 & 3.501 & 0.001 & $0.028 \%$ \\
$\mathrm{r}_{5}$ & 3.500 & 4.176 & 0.676 & $19.314 \%$ \\
\hline
\end{tabular}

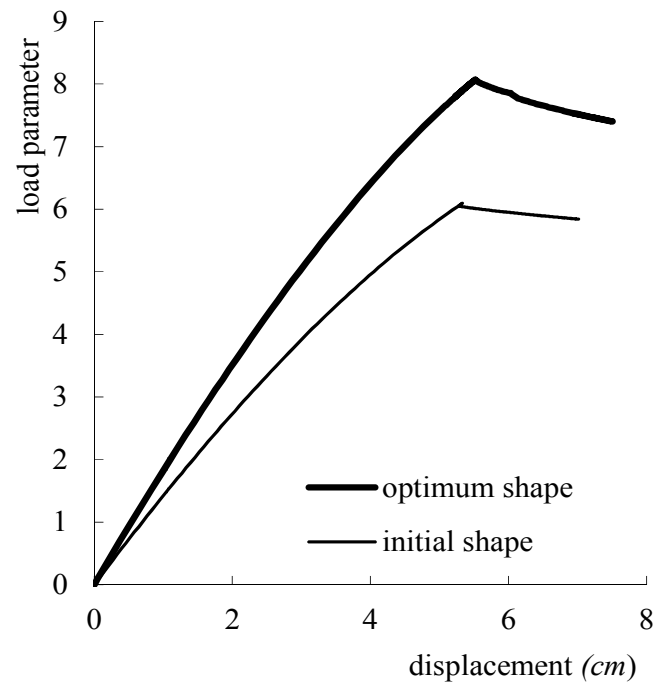

Fig. 7 The load-displacement curves of point "a" due to full area loading case (the position of " $a$ " is shown in Fig. 3).

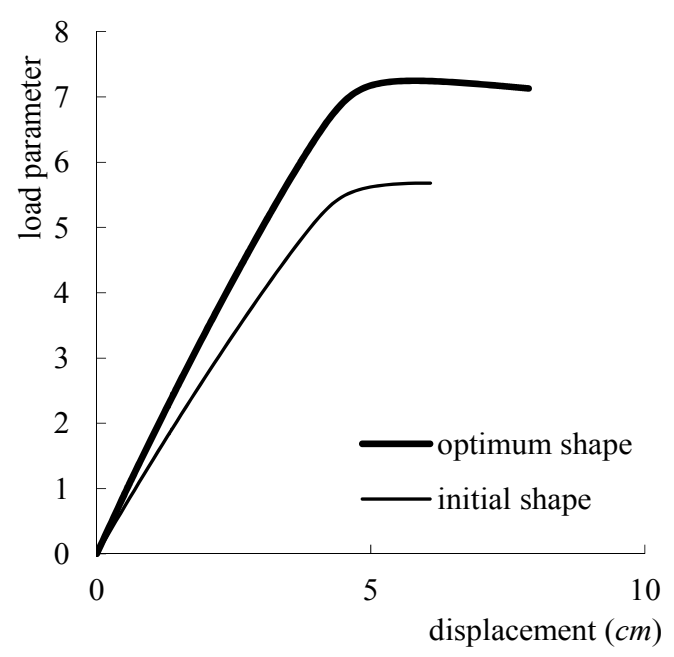

Fig. 8 The load-displacement curves of point "a" due to half area loading case (the position of "a" is shown in Fig. 3).

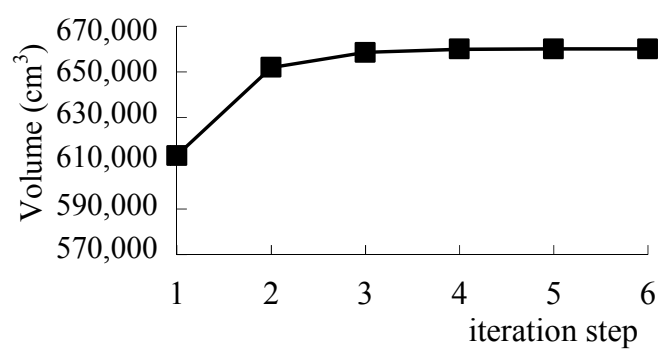

Fig. 9 The change of structural volume.

curves of the imperfection shapes derived from the optimum and initial shapes of Case 1. The imperfection mode is the bifurcation buckling mode of the optimum shape due to the full area loading case, and the ratio of the maximum imperfect point in 
second ring is $R 1: \Delta=1: 0.005$, where $\Delta$ is the maximum of the shape imperfection for $R 1$.

In Fig. 10, the buckling load parameter for the imperfection derived from the optimum shape is bigger than that for the imperfection derived from its initial shape. The displacement at the buckling point for the imperfection derived from the optimum shape is smaller than that of its initial shape. This result indicates that the imperfect shape derived from the optimum shape may buckle at a small deformation, but can support a bigger load comparing to that of its initial shape.

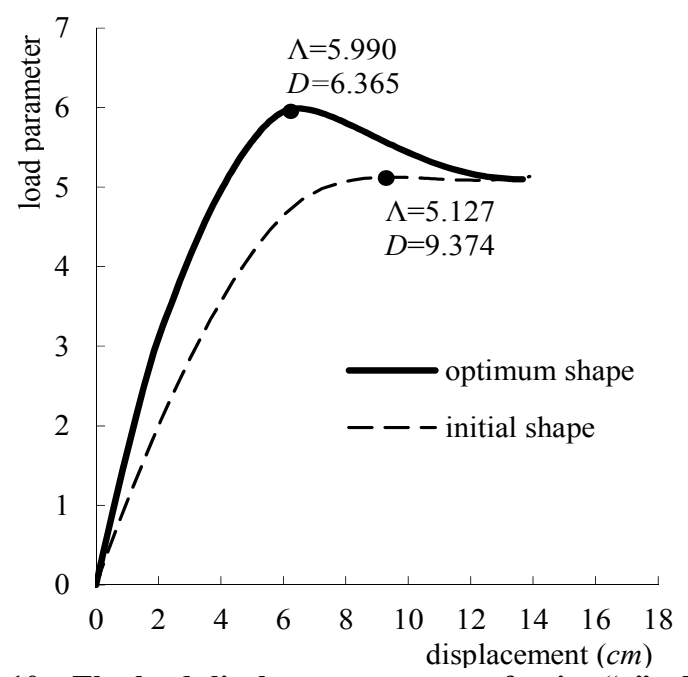

Fig. 10 The load-displacement curves of point "a", shape imperfection for case 1 (full area loading case, point " $a$ " is shown in Fig. 3).

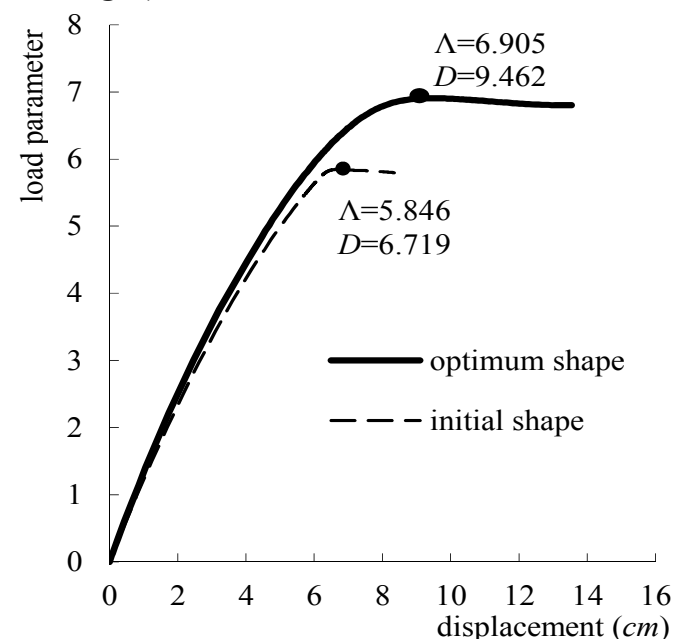

Fig. 11 The load-displacement curves of point "a", shape imperfection for Case 2 (full area loading case, point " $a$ " is shown in Fig. 3).
Fig. 11 shows the load-displacement curves of the imperfect shapes derived from the optimum and initial ones of Case 2. The imperfection mode is the bifurcation buckling mode of the optimum shape due to the full area loading case, and the ratio of the maximum imperfection is the same as Case 1 . It is found that both the load parameter and the displacement at the buckling point for imperfection derived from the optimum shape are bigger than that of its initial shape. This result means that the optimum shape for Case 2 is more stable than that of Case 1. Consequently, an optimum shape with maximum buckling load may not be sensitive to its shape imperfection. Because both the dimensions of shape and member sections are taken as the design parameters in Case 2, the optimization are more efficient than Case 1.

\section{Conclusions}

By mathematical analysis and numerical examples, the author demonstrated that the proposed optimization is effective to find the optimum shape of maximum buckling load. However, this method can find the optima in the vicinity of the initial design parameters, but it may not be efficient to find the global optima in constraint space. The present optimization can determine the member sections automatically and output more information for the structural working design.

The numerical example demonstrated that an optimum shape with maximum buckling load may not be sensitive to its shape imperfection, especially when member section dimensions are taken as design parameters. Nevertheless, mathematic theories of imperfection sensitivity analysis for the present optimization appear as interesting subjects for further researches.

By analyzing the mechanical differences between continuum shells and latticed shells (single layer space frames), the author infers that the two structural systems have different mechanical characteristics, and 
an optimum shape of a latticed shell as well as an orthotropic shell with maximum buckling load may not be sensitive to its geometrical imperfection.

\section{References}

[1] K. Yamamoto, T. Ogawa, M. Fujimoto, C. Lazaro, T. Takeuchi, S.D. Xue, et al., State-of-the-Art for Optimization of Forms and Strength for Reticulated Shells, in: IASS Annual Symposium: IASS-APCS 2012, Seoul, May 21, 2012.

[2] E. Ramm, W.A. Wall, The shell structures_-Prima donna among structures-Efficiency and sensitivity, in: Proceedings of the IASS 2004 Symposium, Montpellier, France, 2004.

[3] R. Reitinger, E. Ramm, Buckling and imperfection sensitivity in the optimization of shell structures, Thin-Walled Structures 23 (1995) 159-177.

[4] M. Ohsaki, K. Ikeda, Imperfection sensitivity analysis of hill-top branching with many symmetric bifurcation points, International Journal of Solids and Structures 43 (2006) 4704-4719.

[5] S.K. Kassegne, J.N. Reddy, Local behavior of discretely stiffened composite plates and cylindrical shells, Composite Structures 41 (1998) 13-26.

[6] S. Yamada, K. Yano, Reduced stiffness buckling criteria of orthotropic cylinders under compression, Transaction of JSCES 2 (2000) 101-106.

[7] C.C. Chao, S.L. Koh, C.T. Sun, Optimization of buckling and yield strengths of laminated composites, AIAA Journal 13 (9) (1975) 1131-1132.

[8] R. Reitinger, E. Ramm, Optimization of geometrically nonlinear buckling sensitive structures, optimization of structural systems and application, in: Third International conference on computer Aided Optimization Design of Structures, Spain, 1993, pp. 525-540.

[9] P.S. Chen, Mamoru Kawaguchi and Masaru Abe, An Application of Inverse Problem Techniques to Spatial Structures, Spatial/Lattice and Tension Structures, Atlanta, Georgia, 1994, pp. 113-122.

[10] P.S. Chen, M. Kawaguchi, Shape of a space frame with maximum buckling load, Journal of Structural and Construction Engineering 489 (1996) 41-46.

[11] E. Riks, An incremental approach to the solution of snapping and buckling problems, Int. J. Solids Structures 15 (1979) 529-551.

[12] E. Riks, Bifurcation and stability, a numerical approach, in: Proceeding of the International Conference on Innovative Methods for Nonlinear Problems, Pineridge Press International Limited, 1984, pp. 313-344.

[13] M. Minoux, Mathematical Programming (Theory and Algorithms), John Wiley and Sons, 1983. 